\title{
Maximum and comparison principles for degenerate elliptic systems and some applications ${ }^{* \dagger}$
}

\author{
Edir Junior Ferreira Leite $\ddagger$ \\ Departamento de Matemática, Universidade Federal de Viçosa, \\ CCE, 36570-900, Viçosa, MG, Brazil
}

\begin{abstract}
In this paper we develop a detailed study on maximum and comparison principles related to the following nonlinear eigenvalue problem

$$
\begin{cases}-\Delta_{p} u=\lambda a(x)|v|^{\beta_{1}-1} v & \text { in } \Omega \\ -\Delta_{q} v=\mu b(x)|u|^{\beta_{2}-1} u & \text { in } \Omega \\ u=v=0 & \text { on } \partial \Omega\end{cases}
$$

where $p, q \in(1, \infty), \beta_{1}, \beta_{2}>0$ satisfy $\beta_{1} \beta_{2}=(p-1)(q-1), \Omega \subset \mathbb{R}^{n}$ is a bounded domain with $C^{2}$-boundary, $a, b \in L^{\infty}(\Omega)$ are given functions, both assumed to be strictly positive on compact subsets of $\Omega$, and $\Delta_{p}$ and $\Delta_{q}$ are quasilinear elliptic operators, stand for $p$-Laplacian and $q$-Laplacian, respectively. We classify all couples $(\lambda, \mu) \in \mathbb{R}^{2}$ such that both the (weak and strong) maximum and comparison principles corresponding to the above system hold in $\Omega$. Explicit lower bounds for principal eigenvalues of this system in terms of the measure of $\Omega$ are also proved. As application, given $\lambda, \mu \geq 0$ we measure explicitly how small has to be $|\Omega|$ so that weak and strong maximum principles associated to the above problem hold in $\Omega$.
\end{abstract}

\section{Introduction and statements}

In this paper we study maximum and comparison principles related to the following system:

$$
\begin{cases}-\Delta_{p} u=\lambda a(x)|v|^{\beta_{1}-1} v & \text { in } \Omega \\ -\Delta_{q} v=\mu b(x)|u|^{\beta_{2}-1} u & \text { in } \Omega \\ u=v=0 & \text { on } \partial \Omega\end{cases}
$$

*AMS Subject Classification 2010: 35B50; 35B51; 35J70; 35J92

${ }^{\dagger}$ Key words: Maximum principles, Comparison principles, Lower bound of eigenvalues

${ }^{\ddagger}$ E-mail addresses: edirjrleite@ufv.br (E.J.F. Leite) 
where $\Omega \subset \mathbb{R}^{n}$ is a bounded domain with $C^{2}$-boundary (not necessarily connected), $p, q \in(1, \infty)$, $a, b \in L^{\infty}(\Omega)$ are given functions satisfying

$$
\underset{x \in \Omega}{\operatorname{essinf}} a(x)>0 \quad \text { and } \quad \underset{x \in \Omega}{\operatorname{essinf}} b(x)>0,
$$

$\beta_{1}, \beta_{2}>0$ with $\beta_{1} \beta_{2}=(p-1)(q-1)$, and $(\lambda, \mu) \in \mathbb{R}^{2}$. The $p$-Laplacian is defined by

$$
\Delta_{p} u:=\operatorname{div}\left(|\nabla u|^{p-2} \nabla u\right)
$$

for any $u \in W_{0}^{1, p}(\Omega)$ with values $\Delta_{p} u \in W^{-1, \frac{p}{p-1}}(\Omega)$, the dual space of $W_{0}^{1, p}(\Omega)$.

Existence, nonexistence and uniqueness of nontrivial solutions to the system (1) have been widely investigated during the three last decades for $p=q=2$ and, more generally, for $p, q \in$ $(1, \infty)$. For $p=q=2$, we refer for instance to [8], [15], [18], 24], 34] and [37, where in particular notions of sub-superlinearity, sub-supercriticality and criticality have been introduced. Still in the first part, the eigenvalue problem, i.e., $\beta_{1} \beta_{2}=1$, was completely studied in [35]. For $p, q \in(1, \infty)$, we refer to [9] when $\beta_{1} \beta_{2}>(p-1)(q-1)$ and [11] when $\beta_{1} \beta_{2}=(p-1)(q-1)$.

The connection between principal eigenvalues and maximum principles have been investigated in [1, 2, 3, 7, 32] for cooperative systems and in [38 for non-cooperative systems (see also [30] for a more complete discussion) and more recently in [26, where system (1) was analyzed in the special case when $p=q=2$, however, instead of $\Delta$, a general second order elliptic operator was considered. For the nonlocal context, we refer to [27].

Here we extend the results of [26] for $p, q>1$, that is, we establish the connection between principal spectral curves for systems (1) and maximum and comparison principles related. For this, we shall present a bit of notation. Note that, given any $f \in L^{\infty}(\Omega)$, there exists a unique weak solution $u \in W_{0}^{1, p}(\Omega)$ of the classical problem

$$
\left\{\begin{aligned}
-\Delta_{p} u & =f(x) & & \text { in } \Omega ; \\
u & =0 & & \text { on } \partial \Omega .
\end{aligned}\right.
$$

Notice that, $u \in C^{1, \alpha}(\bar{\Omega})$ for some $\alpha \in(0,1)$ (see [16, 22, 29, 40]). We denote $X:=\left[C_{0}^{1}(\bar{\Omega})\right]^{2}$, $X_{+}:=\{(u, v) \in X: u \geq 0$ and $v \geq 0$ in $\Omega\}$, and $\stackrel{\circ}{X_{+}}$is the topological interior of $X_{+}$in $X$. Note that, $\stackrel{\circ}{X}_{+}$is nonempty and characterized by $(u, v) \in \stackrel{\circ}{X}_{+}$if, and only if, $(u, v) \in X$ satisfies:

$$
u, v>0 \text { in } \Omega \text { and } \frac{\partial u}{\partial \nu}, \frac{\partial v}{\partial \nu}<0 \text { on } \partial \Omega,
$$

where $\nu \equiv \nu\left(x_{0}\right)$ denotes the exterior unit normal to $\partial \Omega$ at $x_{0} \in \partial \Omega$ (see [11]).

As is well known, the operator $\Delta_{p}$ satisfies the weak maximum principle, that is, for any weak solution $u \in W_{0}^{1, p}(\Omega)$ to

$$
\left\{\begin{aligned}
-\Delta_{p} u & =f(x) & & \text { in } \Omega \\
u & \geq 0 & & \text { on } \partial \Omega
\end{aligned}\right.
$$


with $f \in L^{\infty}(\Omega)$ and $f \geq 0$ in $\Omega$, one has $u \geq 0$ in $\Omega$ and $\Delta_{p}$ also satisfies the strong maximum principle, i.e., moreover $u>0$ in $\Omega$ whenever $f \not \equiv 0$ in $\Omega$ (see [22], 39] and [41]).

Let $(u, v)$ in $W_{0}^{1, p}(\Omega) \times W_{0}^{1, q}(\Omega)$. The weak formulation of the system $(1)$ is given by

$$
\lambda \int_{\Omega} a(x)|v|^{\beta_{1}-1} v w d x=\int_{\Omega}|\nabla u|^{p-2} \nabla u \nabla w d x, \quad \forall w \in C_{0}^{1}(\Omega)
$$

and

$$
\mu \int_{\Omega} b(x)|u|^{\beta_{2}-1} u w d x=\int_{\Omega}|\nabla v|^{q-2} \nabla v \nabla w d x, \quad \forall w \in C_{0}^{1}(\Omega) .
$$

A couple $(\lambda, \mu) \in \mathbb{R}_{+}^{*} \times \mathbb{R}_{+}^{*}=(0, \infty)^{2}$ is said to be an eigenvalue of (1) if the system admits a nontrivial weak solution $(\varphi, \psi)$ in $W_{0}^{1, p}(\Omega) \times W_{0}^{1, q}(\Omega)$ which is called an eigenfunction associated to $(\lambda, \mu)$. We say that $(\lambda, \mu)$ is a principal eigenvalue if admits a positive eigenfunction $(\varphi, \psi)$; i.e., $\varphi$ and $\psi$ are positive in $\Omega$. We also say that $(\lambda, \mu)$ is simple in $\stackrel{\circ}{X}_{+}$if for any eigenfunctions $(\varphi, \psi),(\tilde{\varphi}, \tilde{\psi}) \in \stackrel{\circ}{X}_{+}$, there exists $\rho>0$ such that $\tilde{\varphi}=\rho \varphi$ and $\tilde{\psi}=\rho \mu^{\frac{1}{\beta_{2}}} \psi$ in $\Omega$.

The existence of principal eigenvalues of (1) and some of their qualitative properties were treated in Cuesta and Takác [11]. Namely, they proved that the set formed by these principal eigenvalues is given by the following smooth curve

$$
\mathcal{C}_{1}:=\left\{(\lambda, \mu) \in\left(\mathbb{R}_{+}^{*}\right)^{2}: \lambda^{\frac{1}{\sqrt{\beta_{1}(p-1)}}} \mu^{\frac{1}{\sqrt{\beta_{2}(q-1)}}}=\Lambda^{\prime}\right\}
$$

for some $\Lambda^{\prime}>0$, which satisfies:

(i) $(\lambda, \mu) \in \mathbb{R}_{+} \times \mathbb{R}_{+}$is a principal eigenvalue of the system (1) if, and only if, $(\lambda, \mu) \in \mathcal{C}_{1}$;

(ii) The curve $\mathcal{C}_{1}$ is simple in $\stackrel{\circ}{X}_{+}$, that is, $(\lambda, \mu)$ is simple in $\stackrel{\circ}{X}_{+}$for all $(\lambda, \mu) \in \mathcal{C}_{1}$;

(iii) Let $(\varphi, \psi) \in X$ be an eigenfunction corresponding to $(\lambda, \mu) \in \mathcal{C}_{1}$. Therefore either $(\varphi, \psi) \in$ $\stackrel{\circ}{X}_{+}$or $(-\varphi,-\psi) \in \stackrel{\circ}{X}_{+}$.

Recently, in [28] the author established another properties satisfied by principal curve $\mathcal{C}_{1}$ such as local isolation, monotonicity on $\Omega$ and monotonicity and continuity of the principal eigenvalue with respect to the weight functions $a$ and $b$.

By weak maximum principle, denoted by (WMP), we mean that for any weak solution $(u, v) \in W_{0}^{1, p}(\Omega) \times W_{0}^{1, q}(\Omega)$ of the system

$$
\begin{cases}-\Delta_{p} u=\lambda a(x)|v|^{\beta_{1}-1} v+f(x) & \text { in } \Omega ; \\ -\Delta_{q} v=\mu b(x)|u|^{\beta_{2}-1} u+g(x) & \text { in } \Omega ; \\ u=v=0 & \text { on } \partial \Omega,\end{cases}
$$

with $f, g \in L^{\infty}(\Omega)$ and $f, g \geq 0$ in $\Omega$, verifies $u, v \geq 0$ in $\Omega$. Besides, if at least, $u$ or $v$ is positive in $\Omega$ whenever $f+g \not \equiv 0$ in $\Omega$, we say that the strong maximum principle, denoted by (SMP), corresponding to (1) holds in $\Omega$. When $\lambda, \mu>0$, (SMP) can be rephrased as $u, v>0$ in $\Omega$ 
whenever $f+g \not \equiv 0$ in $\Omega$. In the case that $u>0(v>0)$ in $\Omega$, we get $\frac{\partial}{\partial \nu} u\left(x_{0}\right)<0\left(\frac{\partial}{\partial \nu} v\left(x_{0}\right)<0\right)$ for all $x_{0} \in \partial \Omega$.

We are ready to classify completely in terms of the principal curve $\mathcal{C}_{1}$ the set of couples $(\lambda, \mu) \in \mathbb{R}^{2}$ such that (WMP) and (SMP) hold in $\Omega$.

Namely:

Theorem 1.1. Let $(\lambda, \mu) \in \mathbb{R}^{2}$ and $\mathcal{R}_{1}$ be the open region in the first quadrant below $\mathcal{C}_{1}$. The following assertions are equivalent:

(i) $(\lambda, \mu) \in \overline{\mathcal{R}_{1}} \backslash \mathcal{C}_{1}$;

(ii) (WMP) corresponding to (1) holds in $\Omega$;

(iii) (SMP) corresponding to (1) holds in $\Omega$.

Note that the sets

$$
\mathcal{R}_{1}=\left\{(\lambda, \mu) \in\left(\mathbb{R}_{+}^{*}\right)^{2}: \lambda^{\frac{1}{\sqrt{\beta_{1}(p-1)}}} \mu^{\frac{1}{\sqrt{\beta_{2}(q-1)}}}<\Lambda^{\prime}\right\}
$$

and $\overline{\mathcal{R}_{1}} \backslash \mathcal{C}_{1}=\mathcal{R}_{1} \cup\{(\lambda, 0): \lambda \geq 0\} \cup\{(0, \mu): \mu \geq 0\}$ are unbounded and are depicted in Figure 1.

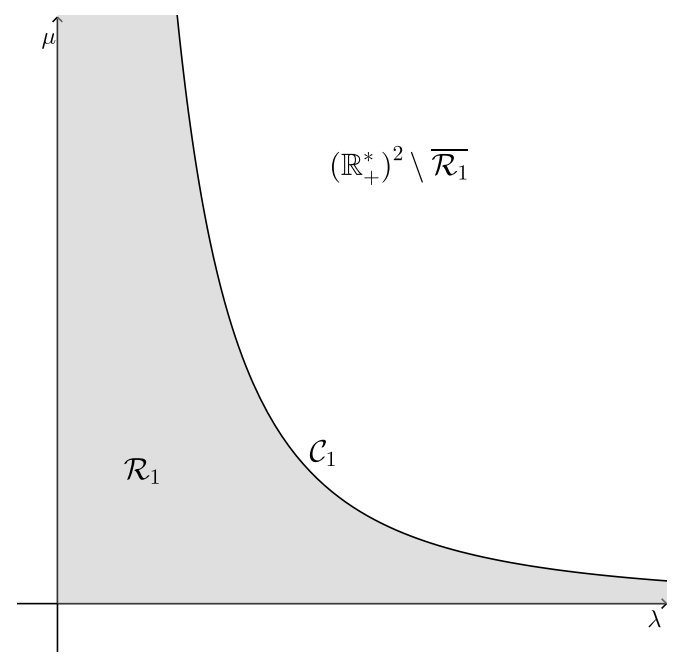

Figure 1: The principal curve $\mathcal{C}_{1}$.

We notice that weak and strong comparison principles, denoted respectively by (WCP) and (SCP), are very important tools for establish the uniqueness and positivity of solutions for elliptic problems, among others, to certain counterparts of (1). We say that (WCP) holds in 
$\Omega$ if, for any weak solutions $(u, v)$ and $(z, w)$ in $W_{0}^{1, p}(\Omega) \times W_{0}^{1, q}(\Omega)$ to the following systems, respectively:

$$
\begin{aligned}
& \begin{cases}-\Delta_{p} u=\lambda a(x)|v|^{\beta_{1}-1} v+f_{1}(x) & \text { in } \Omega ; \\
-\Delta_{q} v=\mu b(x)|u|^{\beta_{2}-1} u+g_{1}(x) & \text { in } \Omega ; \\
u=v=0 & \text { on } \partial \Omega,\end{cases} \\
& \begin{cases}-\Delta_{p} z=\lambda a(x)|w|^{\beta_{1}-1} w+f_{2}(x) & \text { in } \Omega ; \\
-\Delta_{q} w=\mu b(x)|z|^{\beta_{2}-1} z+g_{2}(x) & \text { in } \Omega ; \\
z=w=0 & \text { on } \partial \Omega\end{cases}
\end{aligned}
$$

with $f_{1}, f_{2}, g_{1}, g_{2} \in L^{\infty}(\Omega)$ and $0 \leq f_{1} \leq f_{2}$ and $0 \leq g_{1} \leq g_{2}$ in $\Omega$, one has $u \leq z$ and $v \leq w$ in $\Omega$. If, in addition, at least, $u<z$ or $v<w$ in $\Omega$ whenever $f_{1}+g_{1} \not \equiv f_{2}+g_{2}$ in $\Omega$, we say that (SCP) corresponding to (1) holds in $\Omega$. When $\lambda, \mu>0$, (SCP) in $\Omega$ can be rewritten as $u<z$ and $v<w$ in $\Omega$ whenever $f_{1}+g_{1} \not \equiv f_{2}+g_{2}$ in $\Omega$. In the case that $u<z(v<w)$ in $\Omega$, we clearly have $\frac{\partial}{\partial \nu} u\left(x_{0}\right)>\frac{\partial}{\partial \nu} z\left(x_{0}\right)\left(\frac{\partial}{\partial \nu} v\left(x_{0}\right)>\frac{\partial}{\partial \nu} w\left(x_{0}\right)\right)$ for all $x_{0} \in \partial \Omega$.

Our next theorem characterizes completely the (WCP) and (SCP) corresponding to (1) in terms of the smooth curve $\mathcal{C}_{1}$. Precisely:

Theorem 1.2. Let $\mathcal{R}_{1}$ be as in Theorem 1.1 and $(\lambda, \mu) \in \mathbb{R}^{2}$. The following assertions are equivalent:

(i) $(\lambda, \mu) \in \overline{\mathcal{R}_{1}} \backslash \mathcal{C}_{1}$;

(ii) (WCP) corresponding to (1) holds in $\Omega$;

(iii) (SCP) corresponding to (1) holds in $\Omega$.

The validity of weak and strong comparison principles for problems involving the $p$-Laplacian operator is usually very delicate (see [10, 11, 12, 13, 14, 19, 20, 21, 22, 23, 36, 39]). For example, the operator $\Delta_{p}$ satisfies the strong comparison principle only under the additional condition zero Dirichlet boundary values on $\partial \Omega$. So, we need assume the same condition on the (WMP), (SMP), (WCP) and (SCP) associated to the problem (1). Moreover, we prove the (WCP) and (SCP) under the following additional condition: $f_{1} \geq 0$ and $g_{1} \geq 0$ in $\Omega$, when $(\lambda, \mu) \in \mathcal{R}_{1}$.

Now, we characterize when such (WMP) (or (SMP)) corresponding to (1) is satisfied in domains $\Omega$ of small Lebesgue measure. For this, we shall obtain an explicit lower estimate of $\Lambda^{\prime}$ in terms of the Lebesque measure of $\Omega$.

In Theorem 2.6 of [5], Berestycki, Nirenberg and Varadhan established a lower estimate for principal eigenvalues corresponding to the problem, in the scalar context, involving linear second order elliptic operators. In Theorem 5.1 of [31, López-Gómez obtained an explicit lower estimate for such principal eigenvalues. Later, in Theorem 10.1 of [6] Cano-Casanova and López-Gómez extended this result for mixed boundary conditions. The key tool for the proof of the Theorem 2.6 of [5] is an ABP estimate for second order uniformly elliptic operators while in proofs of the Theorems 5.1 of [31] and 10.1 of [6] is used in a crucial way the celebrated Faber-Krahn inequality 
of Faber [17] and Krahn [25]. Recently, in [28] the author obtained an explicit lower bounds for principal eigenvalues of the system (1) via Faber-Krahn inequality for the first eigenvalue of $-\Delta_{p}$. Here, we will use an ABP estimate related to $p$-Laplacian operator with explicit constant (see Theorem 3 of [4]). Namely:

Theorem 1.3. Let $a, b \in L^{\infty}(\Omega) \cap C(\Omega)$ and $\mathcal{C}_{1}$ be the principal curve associated to (1). Then

$$
\Lambda^{\prime} \geq \frac{|\Omega|^{-\left(\frac{1}{n s}+\frac{1}{n r}\right)}}{c(n, p)^{\frac{\beta_{2}}{s}} c(n, q)^{\frac{\beta_{1}}{r}} d^{\frac{\beta_{2}}{s}+\frac{\beta_{1}}{r}}\|a\|_{L^{\infty}(\Omega)}^{\frac{1}{r}}\|b\|_{L^{\infty}(\Omega)}^{\frac{1}{s}}},
$$

where $d:=\operatorname{diam}(\Omega), r:=\sqrt{\beta_{1}(p-1)}, s:=\sqrt{\beta_{2}(q-1)},|\cdot|$ stands for the Lebesgue measure of $\mathbb{R}^{n}$,

$$
c(n, p):=\left(n \min \{1, p-1\}\left|B_{1}\right|^{\frac{1}{n}}\right)^{-\frac{1}{p-1}}
$$

and $B_{1}$ is the unit ball of $\mathbb{R}^{n}$. In particular,

$$
\lim _{|\Omega| \downarrow 0} \Lambda^{\prime}=+\infty
$$

Note that we get an explicit lower estimate of $\Lambda^{\prime}$ in terms of the Lebesque measure of $\Omega$, diameter of $\Omega$, explicit constants $c(n, p), c(n, q)$ and the weighted functions $a, b \in L^{\infty}(\Omega) \cap C(\Omega)$. As an interesting consequence of Theorems 1.1 and 1.3 , we obtain the following characterization of maximum principles:

Theorem 1.4. Let $r, s, c(n, p)$ and $c(n, q)$ be as in Theorem 1.3 and $a, b \in L^{\infty}(\Omega) \cap C(\Omega)$. Define $d:=\operatorname{diam}(\Omega)$ and

$$
\eta:=\frac{1}{\left[\lambda^{\frac{1}{r}} \mu^{\frac{1}{s}}\left(c(n, p)^{\frac{\beta_{2}}{s}} c(n, q)^{\frac{\beta_{1}}{r}} d^{\frac{\beta_{2}}{s}+\frac{\beta_{1}}{r}}\|a\|_{L^{\infty}(\Omega)}^{\frac{1}{r}}\|b\|_{L^{\infty}(\Omega)}^{\frac{1}{s}}\right)\right]^{\frac{n r s}{r+s}}} .
$$

The following assertions are equivalent:

(i) $\lambda \geq 0$ and $\mu \geq 0$;

(ii) (WMP) corresponding to (1) holds in $\Omega$ provided that $|\Omega|<\eta$;

(iii) (SMP) corresponding to (1) holds in $\Omega$ provided that $|\Omega|<\eta$.

Finally, as application of Theorems 1.1 and 1.2 , we present a characterization in terms of the principal curve $\mathcal{C}_{1}$ the set of couples $(\lambda, \mu) \in \mathbb{R}^{2}$ such that the system (3) admits a unique weak nonnegative solution $(u, v)$ for any pair $(f, g) \in\left(L^{\infty}(\Omega)\right)^{2}$ of nonnegative functions. Precisely, we have: 
Theorem 1.5. Let $\mathcal{R}_{1}$ be as in Theorem 1.1 and $(\lambda, \mu) \in \mathbb{R}^{2}$. Then, the couple $(\lambda, \mu) \in \overline{\mathcal{R}_{1}} \backslash \mathcal{C}_{1}$ if, and only if, the system (3) admits a unique weak solution $(u, v) \in W_{0}^{1, p}(\Omega) \times W_{0}^{1, q}(\Omega)$ and this satisfies $u, v \geq 0$ in $\Omega$ for any pair $(f, g) \in\left(L^{\infty}(\Omega)\right)^{2}$ of nonnegative functions. Moreover, $(u, v) \in\left(C^{1, \alpha}(\bar{\Omega})\right)^{2}$ for some $\alpha \in(0,1)$.

The rest of paper is organized into five sections. In Section 2 we show Theorem 1.1 by mean of maximum and comparison principles and Hopf's lemma related to the $p$-Laplacian operator. In Section 3 we characterize weak and strong comparison principles associated to the system (1) stated in Theorem 1.2 by using Theorem 1.1 as a key tool. In Section 4 we get an explicit lower estimate of $\Lambda^{\prime}$ in terms of the Lebesque measure of $\Omega$ stated Theorem 1.3 . The characterization of maximum principle in domains $\Omega$ of small Lebesgue measure stated in Theorem 1.4 is established in Section 5. Finally, in Section 6 we prove Theorem 1.5 by using Theorems 1.1 and 1.2 .

\section{Proof of Theorem 1.1}

In this section we prove Theorem 1.1, which classify completely the couples $(\lambda, \mu) \in \mathbb{R}^{2}$ such that (WMP) and (SMP) hold in $\Omega$. Notice that it suffices to show only that (i) $\Leftrightarrow$ (ii). In this case, we clearly have (ii) $\Leftrightarrow$ (iii). In fact, it is obvious that (SMP) in $\Omega$ implies (WMP) in $\Omega$. Conversely, assume that (WMP) holds in $\Omega$ and let $(u, v)$ be a weak solution of the system (3) with $f, g \in L^{\infty}(\Omega), f, g \geq 0$ in $\Omega$ and $f+g \not \equiv 0$ in $\Omega$. Thus, $u, v \geq 0$ in $\Omega$ and, by (i), we obtain $\lambda, \mu \geq 0$. Then, the conclusion of (SMP) follows, since $\Delta_{p}$ satisfies the strong maximum principle (see [22, 39, 41]).

In order to proof that (WMP) in $\Omega$ leads to $(\lambda, \mu) \in \overline{\mathcal{R}_{1}} \backslash \mathcal{C}_{1}$, assume instead that $(\lambda, \mu) \notin$ $\overline{\mathcal{R}_{1}} \backslash \mathcal{C}_{1}$. Let $(\lambda, \mu) \in \mathcal{C}_{1}$ and $(\tilde{\varphi}, \tilde{\psi})$ be a positive eigenfunction associated to $(\lambda, \mu)$. Then, $(-\tilde{\varphi},-\tilde{\psi})$ is a negative eigenfunction associated to $(\lambda, \mu)$ and so (WMP) fails in $\Omega$.

Assume now that $(\lambda, \mu) \in \mathbb{R}^{2}$ is a fixed couple outside of $\overline{\mathcal{R}_{1}}$. If $(\lambda, \mu) \in\left(\mathbb{R}_{+}^{*}\right)^{2}$, we get $\lambda>\lambda_{1}$ and $\mu>\mu_{1}$, where $\left(\lambda_{1}, \mu_{1}\right)$ is a principal eigenvalue of 11 with $\frac{\mu}{\lambda}=\frac{\mu_{1}}{\lambda_{1}}$. Denote by $(\varphi, \psi)$ a positive eigenfunction corresponding to $\left(\lambda_{1}, \mu_{1}\right)$. Thus, we derive

$$
\left\{\begin{aligned}
-\Delta_{p}(-\varphi)-\lambda a(x)|-\psi|^{\beta_{1}-1}(-\psi) & =-\lambda_{1} a(x) \psi^{\beta_{1}}+\lambda a(x) \psi^{\beta_{1}} \\
& =\left(\lambda-\lambda_{1}\right) a(x) \psi^{\beta_{1}} \geq(\not \equiv) 0 \quad \text { in } \Omega ; \\
-\Delta_{q}(-\psi)-\mu b(x)|-\varphi|^{\beta_{2}-1}(-\varphi) & =-\mu_{1} b(x) \varphi^{\beta_{2}}+\mu b(x) \varphi^{\beta_{2}} \\
& =\left(\mu-\mu_{1}\right) b(x) \varphi^{\beta_{2}} \geq(\not \equiv) 0 \quad \text { in } \Omega
\end{aligned}\right.
$$

and $-\varphi=0=-\psi$ on $\partial \Omega$. Since, $-\varphi,-\psi<0$ in $\Omega$, (WMP) doesn't hold in $\Omega$.

Now, suppose that $\lambda<0$. Then, there exists $\left(\lambda_{1}, \mu_{1}\right) \in \mathcal{C}_{1}$ with $\lambda_{1}>0$ small enough (and so $\mu_{1}>0$ large enough) so that $\lambda<-\lambda_{1}$ and $\mu>-\mu_{1}$. Then, we get 


$$
\left\{\begin{array}{lll}
-\Delta_{p}(-\varphi)-\lambda a(x) \psi^{\beta_{1}} & =-\lambda_{1} a(x) \psi^{\beta_{1}}-\lambda a(x) \psi^{\beta_{1}} & \\
& =-\left(\lambda+\lambda_{1}\right) a(x) \psi^{\beta_{1}} \geq(\not \equiv) 0 \quad \text { in } \Omega ; \\
-\Delta_{q} \psi-\mu b(x)|-\varphi|^{\beta_{2}-1}(-\varphi) & =\mu_{1} b(x) \varphi^{\beta_{2}}+\mu b(x) \varphi^{\beta_{2}} & \\
& =\left(\mu+\mu_{1}\right) b(x) \varphi^{\beta_{2}} \geq(\not \equiv) 0 \quad \text { in } \Omega
\end{array}\right.
$$

and $-\varphi=0=\psi$ on $\partial \Omega$. However, $-\varphi<0$ in $\Omega$ and so (WMP) fails in $\Omega$.

For the remaining case $\lambda \geq 0$ and $\mu<0$, there exists $\left(\lambda_{1}, \mu_{1}\right) \in \mathcal{C}_{1}$ with $\lambda_{1}>0$ large enough (and so $\mu_{1}>0$ small enough) so that $\lambda>-\lambda_{1}$ and $\mu<-\mu_{1}$. Therefore,

$$
\left\{\begin{array}{rll}
-\Delta_{p} \varphi-\lambda a(x)|-\psi|^{\beta_{1}-1}(-\psi) & =\lambda_{1} a(x) \psi^{\beta_{1}}+\lambda a(x) \psi^{\beta_{1}} & \\
& =\left(\lambda+\lambda_{1}\right) a(x) \psi^{\beta_{1}} \geq(\not \equiv) 0 \quad \text { in } \Omega ; \\
-\Delta_{q}(-\psi)-\mu b(x) \varphi^{\beta_{2}} & =-\mu_{1} b(x) \varphi^{\beta_{2}}-\mu b(x) \varphi^{\beta_{2}} \\
& =-\left(\mu+\mu_{1}\right) b(x) \varphi^{\beta_{2}} \geq(\not \equiv) 0 \quad \text { in } \Omega
\end{array}\right.
$$

and $\varphi=0=-\psi$ on $\partial \Omega$. But, $-\psi<0$ in $\Omega$ and so again (WMP) fails in $\Omega$.

Conversely, we next show that (WMP) holds in $\Omega$ for any couple $(\lambda, \mu) \in \overline{\mathcal{R}_{1}} \backslash \mathcal{C}_{1}$. Since $\Delta_{p}$ and $\Delta_{q}$ satisfies weak maximum principle in $\Omega$, we have (WMP) holds in $\Omega$ if either $\lambda=0$ and $\mu \geq 0$ or $\lambda \geq 0$ and $\mu=0$. Now let $(\lambda, \mu) \in \mathcal{R}_{1}$. Let $(u, v)$ be a weak solution of the system (3). Note that $\lambda<\lambda_{1}$ and $\mu<\mu_{1}$, where $\left(\lambda_{1}, \mu_{1}\right)$ is a principal eigenvalue of (1) with $\frac{\mu}{\lambda}=\frac{\mu_{1}}{\lambda_{1}}$. Let $(\varphi, \psi)$ be a positive eigenfunction associated to $\left(\lambda_{1}, \mu_{1}\right)$. Arguing by contradiction, assume that $u$ or $v$ is negative somewhere in $\Omega$. Then, by strong comparison principle and Hopf's lemma for the $p$-Laplacian (see [22, 39, 41]), there exists some $\gamma>0$ such that

$$
-u \leq \gamma \varphi \text { and }-v \leq \gamma^{\omega} \psi \text { in } \Omega,
$$

where $\omega:=\frac{p-1}{\beta_{1}}$. Let $\bar{\gamma}$ be the minimum of such $\gamma^{\prime} s$. Thus, $\bar{\gamma}>0$. Since $\lambda<\lambda_{1}$ and $\mu<\mu_{1}$, we derive

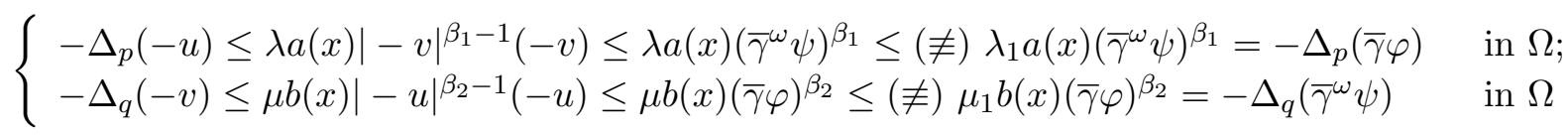

and $\bar{\gamma} \varphi=-u=\bar{\gamma}^{\omega} \psi=-v=0$ on $\partial \Omega$. It follows from the strong comparison principle to each above equation (see Theorem A.1 of [11]) that $-u<\bar{\gamma} \varphi$ and $-v<\bar{\gamma}^{\omega} \psi$ in $\Omega$. Thus, we can find $0<\varepsilon<1$ such that $-u \leq \varepsilon \bar{\gamma} \varphi$ and $-v \leq(\varepsilon \bar{\gamma})^{\omega} \psi$ in $\Omega$, contradicting the definition of $\bar{\gamma}$. Then, $u, v \geq 0$ in $\Omega$. Hence, we complete the wished proof of theorem.

\section{Proof of Theorem 1.2}

In this section we establish the characterization of (WCP) and (SCP) in terms of the principal curve $\mathcal{C}_{1}$ as stated in Theorem 1.2. Using strong comparison principle (see Theorem A.1 of [11]) and arguing in a similar way as in the proof of Theorem 1.1, we see that it suffices to show only that (i) $\Leftrightarrow$ (ii). 
(ii) $\Rightarrow$ (i) Taking $u, v \equiv 0$ in $\Omega$ (and so $f_{1}, f_{2} \equiv 0$ in $\Omega$ ), we have (WCP) in $\Omega$ implies (WMP) in $\Omega$. Then, by Theorem 1.1, (WCP) in $\Omega$ implies $(\lambda, \mu) \in \overline{\mathcal{R}_{1}} \backslash \mathcal{C}_{1}$.

(i) $\Rightarrow$ (ii) Consider $(\lambda, \mu) \in \overline{\mathcal{R}_{1}} \backslash \mathcal{C}_{1}$. Since $\Delta_{p}$ satisfies weak maximum and comparison principles in $\Omega$, the conclusion is direct in the cases that $\lambda=0$ or $\mu=0$. Thus, it suffices to consider $(\lambda, \mu) \in \mathcal{R}_{1}$. In this case, since $(u, v)$ is a weak solution of the problem (4), by Theorem 1.1. if $f_{1}, g_{1} \equiv 0$ in $\Omega$ we have $u, v \equiv 0$ in $\Omega$ and if $f_{1}+g_{1} \not \equiv 0$ in $\Omega$, we get $u, v>0$ in $\Omega$. Note that, if $f_{1}, g_{1} \equiv 0$ in $\Omega$, then the conclusion follows readily from (WMP) in $\Omega$. Assume then $f_{1}+g_{1} \not \equiv 0$ in $\Omega$ (and so $u, v>0$ in $\Omega$ ). Thus, we also have $f_{2}+g_{2} \not \equiv 0$ in $\Omega$. Therefore, since $(z, w)$ is a weak solution of (5), by (SMP), we have $z, w>0$ in $\Omega$.

To finish the proof of (WCP), it suffices to show that $u \leq z$ in $\Omega$. This follows directly by using the fact that $\Delta_{q}$ satisfies weak comparison principle in $\Omega$ (see [39]). Assume by contradiction that $u>z$ somewhere in $\Omega$. In this case, the set $\Gamma:=\left\{\gamma>0: z>\gamma u\right.$ and $w>\gamma^{\omega} v$ in $\left.\Omega\right\}$, where $\omega:=\frac{p-1}{\beta_{1}}$, is nonempty by Hopf's Lemma (see [41]) and is also upper bounded. Set $\bar{\gamma}:=\sup \Gamma>0$. Notice that $z \geq \bar{\gamma} u$ and $w \geq \bar{\gamma}^{\omega} v$ in $\Omega$. Note also that the statement of contradiction leads to $\bar{\gamma}<1$. Thus, since $f_{1}+g_{1} \not \equiv 0$ and $f_{2}+g_{2} \not \equiv 0$ in $\Omega$, we derive

$$
\begin{cases}-\Delta_{p}(\bar{\gamma} u)=\lambda a(x)\left(\bar{\gamma}^{\omega} v\right)^{\beta_{1}}+\bar{\gamma}^{p-1} f_{1} \leq(\not \equiv) \lambda a(x) w^{\beta_{1}}+f_{2}=-\Delta_{p}(z) & \text { in } \Omega \\ -\Delta_{q}\left(\bar{\gamma}^{\omega} v\right)=\mu b(x)(\bar{\gamma} u)^{\beta_{2}}+\bar{\gamma}^{\beta_{2}} g_{1} \leq(\not \equiv) \mu b(x) z^{\beta_{2}}+g_{2}=-\Delta_{q}(w) & \text { in } \Omega\end{cases}
$$

and $\bar{\gamma} u=z=\bar{\gamma}^{\omega} v=w=0$ on $\partial \Omega$. It follows from the strong comparison principle to each above equation (see Theorem A.1 of [11]) that $z>\bar{\gamma} u$ and $w>\bar{\gamma}^{\omega} v$ in $\Omega$. Thus, we can find $0<\varepsilon<1$ such that $z \geq(\bar{\gamma}+\varepsilon) u$ and $w \geq(\bar{\gamma}+\varepsilon)^{\omega} v$ in $\Omega$, contradicting the definition of $\bar{\gamma}$. This concludes the desired proof.

\section{Proof of Theorem 1.3}

Let $a, b \in L^{\infty}(\Omega) \cap C(\Omega),\left(\lambda_{1}, \mu_{1}\right) \in \mathcal{C}_{1}$ and $(\varphi, \psi) \in X$ be a positive eigenfunction of the problem (1) corresponding to $\left(\lambda_{1}, \mu_{1}\right)$. Since $\lambda_{1} a \varphi^{\beta_{1}}, \mu_{1} b \psi^{\beta_{2}} \in C(\Omega)$, by Theorem 1.8 of [33], we have $(\varphi, \psi)$ is a viscosity subsolution of the system (1). Thus, applying the ABP estimate for the $p$-Laplacian (see Theorem 3 of [4]) to the first equation of (1), we have

$$
\|\varphi\|_{L^{\infty}(\Omega)}=\sup _{\Omega} \varphi \leq c(n, p) d \lambda_{1}^{\frac{1}{p-1}}\|a\|_{L^{\infty}(\Omega)}^{\frac{1}{p-1}}\|\psi\|_{L^{\infty}(\Omega)}^{\frac{\beta_{1}}{p-1}}|\Omega|^{\frac{1}{n(p-1)}}
$$

and for the $q$-Laplacian to the second equation of (1), we obtain

$$
\|\psi\|_{L^{\infty}(\Omega)} \leq c(n, q) d \mu_{1}^{\frac{1}{q-1}}\|b\|_{L^{\infty}(\Omega)}^{\frac{1}{q-1}}\|\varphi\|_{L^{\infty}(\Omega)}^{\frac{\beta_{2}}{q-1}}|\Omega|^{\frac{1}{n(q-1)}}
$$

Therefore, joining inequalities (7) and (8) and using that

$$
\beta_{1} \beta_{2}=(p-1)(q-1) \text { and } \lambda_{1}^{\frac{1}{r}} \mu_{1}^{\frac{1}{s}}=\Lambda^{\prime},
$$

we finally derive (6) and conclude the proof. 


\section{Proof of Theorem 1.4}

By Theorem 1.1, the necessity of (i) and the equivalence between (WMP) and (SMP) follow directly. Thus, it suffices to prove that the assertion (i) implies (ii).

Assume that $\lambda \geq 0$ and $\mu \geq 0$. If either $\lambda=0$ or $\mu=0$, then by Theorem 1.1, the desired (WMP) follow.

Finally, assume now that $\lambda>0$ and $\mu>0$. We consider the positive constant $\eta$ given by

$$
\eta:=\frac{1}{\left[\lambda^{\frac{1}{r}} \mu^{\frac{1}{s}}\left(c(n, p)^{\frac{\beta_{2}}{s}} c(n, q)^{\frac{\beta_{1}}{r}} d^{\frac{\beta_{2}}{s}+\frac{\beta_{1}}{r}}\|a\|_{L^{\infty}(\Omega)}^{\frac{1}{r}}\|b\|_{L^{\infty}(\Omega)}^{\frac{1}{s}}\right)\right]^{\frac{n r s}{r+s}}},
$$

where $d:=\operatorname{diam}(\Omega), r:=\sqrt{\beta_{1}(p-1)}, s:=\sqrt{\beta_{2}(q-1)}$ and $c(n, p)$ and $c(n, q)$ are the explicit constants of ABP estimate for the $p$-Laplacian and $q$-Laplacian, respectively. Then, by using the estimate (6) of Theorem 1.3 , we obtain

$$
\Lambda^{\prime} \geq \frac{|\Omega|^{-\left(\frac{1}{n s}+\frac{1}{n r}\right)}}{c(n, p)^{\frac{\beta_{2}}{s}} c(n, q)^{\frac{\beta_{1}}{r}} d^{\frac{\beta_{2}}{s}+\frac{\beta_{1}}{r}}\|a\|_{L^{\infty}(\Omega)}^{\frac{1}{r}}\|b\|_{L^{\infty}(\Omega)}^{\frac{1}{s}}}>\lambda^{\frac{1}{r}} \mu^{\frac{1}{s}}
$$

whenever $|\Omega|<\eta$. Therefore, we get $(\lambda, \mu) \in \mathcal{R}_{1}$ for such domains and so, by Theorem 1.1 the assertion (ii) holds. This concludes the proof of theorem.

\section{Proof of Theorem 1.5}

Let $(\lambda, \mu) \in \overline{\mathcal{R}_{1}} \backslash \mathcal{C}_{1}$ and $f, g \in L^{\infty}(\Omega)$ such that $f, g \geq 0$ in $\Omega$. If either $\lambda=0$ or $\mu=0$, then clearly, by existence and uniqueness of weak solution for the problem (2), the system (3) admits a unique weak solution $(u, v) \in W_{0}^{1, p}(\Omega) \times W_{0}^{1, q}(\Omega)$. Applying the weak maximum principle to each equation of (3), we get $u, v \geq 0$ in $\Omega$.

Assume now that $(\lambda, \mu) \in \mathcal{R}_{1}$. Then, by Theorem 3.1 of [11], the system (3) admits a unique weak solution $(u, v) \in X_{+}$. Let $(z, w) \in W_{0}^{1, p}(\Omega) \times W_{0}^{1, q}(\Omega)$ be a weak solution of the system (3). So, by (WCP), $u=z$ and $v=w$ in $\Omega$. Therefore, the system (3) admits a unique weak solution in $W_{0}^{1, p}(\Omega) \times W_{0}^{1, q}(\Omega)$ and this is nonnegative.

Conversely, assume that $(\lambda, \mu) \in \mathbb{R}^{2}$ and the problem (3) admits a unique weak solution $(u, v) \in W_{0}^{1, p}(\Omega) \times W_{0}^{1, q}(\Omega)$ and that this satisfies $u, v \geq 0$ in $\Omega$ for any pair $(f, g) \in\left(L^{\infty}(\Omega)\right)^{2}$ of nonnegative functions. Thus, (WMP) associated to (1) holds in $\Omega$ and so by Theorem 1.1 . we have $(\lambda, \mu) \in \overline{\mathcal{R}_{1}} \backslash \mathcal{C}_{1}$. This ends the proof. 


\section{References}

[1] B. Alziary, J. Fleckinger, M.-H. Lécureux - Principal eigenvalue and Maximum principle for some elliptic systems defined on general domains with refined Dirichlet boundary condition, Comm. Math. Anal. 7 (2009), 1-11.

[2] H. Amann - Maximum principles and principal eigenvalues, in 10 Mathematical Essays on Approximation in Analysis and Topology, (J. Ferrera, J. López-Gómez and F. R. Ruiz del Portal eds.), pp. 1-60, Elsevier, Amsterdam, 2005.

[3] I. Anton, J. López-Gómez - Principal eigenvalue and maximum principle for cooperative periodic-parabolic systems, Nonlinear Analysis 178 (2019), 152-189.

[4] R. Argiolas, F. Charro, I. Peral - On the Aleksandrov-Bakel'man-Pucci Estimate for Some Elliptic and Parabolic Nonlinear Operators, Arch. Rational Mech. Anal. 202 (2011), 875917.

[5] H. Berestycki, L. Nirenberg, S.R.S. Varadhan - The principal eigenvalue and maximum principle for second order elliptic operators in general domains, Comm. pure Appl. Math. (1994), 47-92.

[6] S. Cano-Casanova, J. López-Gómez - Properties of the principal eigenvalues of a general class of nonclassical mixed boundary value problems, J. Diff. Eq. 178 (2002), 123-211.

[7] P.A. Caudevilla, J. López-Gómez - Asymptotic behaviour of principal eigenvalues for a class of cooperative systems, J. Diff. Eq. 244 (2008), 1093-1113.

[8] Ph. Clément, D. G. de Figueiredo, E. Mitidieri - Positive solutions of semilinear elliptic systems, Comm. in PDE 17 (1992), 923-940.

[9] Ph. Clément, R.F. Manásevich, E. Mitidieri - Positive solutions for a quasilinear system via blow up, Comm. P.D.E., 18 (1993), 2071-2106.

[10] M. Cuesta, P. Takác - A strong comparison principle for the Dirichlet p-Laplacian, in Reaction Diffusion Systems, (G. Caristi and E. Mitidieri, Eds.). Lecture Notes in Pure and Applied Mathematics, 194. Marcel Dekker (1997).

[11] M. Cuesta, P. Takác - Nonlinear eigenvalue problems for degenerate elliptic systems, Differ. Integral Equations 23 (2010), 1117-1138.

[12] L. Damascelli - Comparison theorems for some quasilinear degenerate elliptic operators and applications to symmetry and monotonicity results, Ann. Inst. H. Poincaré. Analyse non linéaire 15 (1998), 493-516.

[13] L. Damascelli, B. Sciunzi - Regularity, monotonicity and symmetry of positive solutions of m-Laplace equations, J. Differential Equations 206 (2004), 483-515. 
[14] L. Damascelli, B. Sciunzi - Harnack inequalities, maximum and comparison principles, and regularity of positive solutions of $m$-Laplace equations, Calc. Var. Partial Differential Equations 25 (2006), 139-159.

[15] D. G. de Figueiredo, P. L. Felmer - On superquadratic elliptic systems, Trans. Amer. Math. Soc. 343 (1994), 99-116.

[16] DE. DiBenedetto - $C^{1+\alpha}$ local regularity of weak solutions of degenerate elliptic equations, Nonlinear Anal. 7 (1983), 827-850.

[17] G. Faber - Beweis, dass unter allen homogenen Membranen von gleicher Fläche und gleicher Spannung die kreisförmige den tiefsten Grundton gibt (in German), Sitzungberichte der mathematisch-physikalischen Klasse der Bayerischen Akademie der Wissenschaften zu München Jahrgang (1923), 169-172.

[18] P. Felmer, S. Martínez - Existence and uniqueness of positive solutions to certain differential systems, Adv. Differential Equations 4 (1998), 575-593.

[19] J. Fleckinger-Pellé, J. P. Gossez, P. Takác, F. de Thélin - Nonexistence of solutions and an anti-maximum principle for cooperative systems with the p-Laplacian, Math. Nachr. 194 (1998), 49-78.

[20] J. Fleckinger-Pellé, J. Hernández, P. Takác, F. de Thélin - Uniqueness and positivity for solutions of equations with the p-Laplacian. Reaction diffusion systems (Trieste, 1995), 141155, Lecture Notes in Pure and Appl. Math., 194, Dekker, New York, 1998.

[21] J. Fleckinger-Pellé, P. Takác - Uniqueness of positive solutions for nonlinear cooperative systems with the p-Laplacian, Indiana Univ. Math. J. 43 (1994), 1227-1253.

[22] J. García-Melián, J. C. Sabina de Lis - Maximum and comparison principles for operators involving the p-Laplacian, J. Math. Anal. Appl. 218 (1998), 49-65.

[23] M. Guedda, L. Veron - Quasilinear elliptic equations involving critical Sobolev exponents, Nonlinear Anal. 13 (1989), 879-902.

[24] J. Hulshof, R. C. A. M. van der Vorst - Differential systems with strongly indefinite variational structure, J. Funct. Analysis, 114 (1993), 32-58.

[25] E. Krahn - Über eine von Rayleigh formulierte Minimaleigenschaft des Kreises, Math. Ann. 91 (1925), 97-100.

[26] E. J. F. Leite, M. Montenegro - Maximum and comparison principles to Lane-Emden systems, Journal of the London Mathematical Society 101 (2020), 23-42.

[27] E. J. F. Leite, M. Montenegro - Principal curves to nonlocal Lane-Emden systems and related maximum principles, Calc. Var. Partial Differential Equations 59 (2020), 118. 
[28] E. J. F. Leite - On the principal eigenvalues of the degenerate elliptic systems, Electronic Journal of Qualitative Theory of Differential Equations 40 (2020), 1-15.

[29] G. Lieberman - Boundary regularity for solutions of degenerate elliptic equations, Non-linear Anal. 12 (1988), 1203-1219.

[30] J. López-Gómez - Linear Second Order Elliptic Operators, World Scientific, Singapore, 2013.

[31] J. López-Gómez - The maximum principle and the existence of principal eigenvalue for some linear weighted boundary value problems, J. Diff. Eq. 127 (1996), 263-294.

[32] J. López-Gómez, M. Molina-Meyer - The maximum principle for cooperative weakly coupled elliptic systems and some applications, Diff. Int. Equations 7 (1994), 383-398.

[33] M. Medina, P. Ochoa - On viscosity and weak solutions for non-homogeneous p-Laplace equations, Adv. Nonlinear Anal. 8 (2019), 468-481.

[34] E. Mitidieri - A Rellich type identity and applications, Comm. Partial Differential Equations 18 (1993), 125-151.

[35] M. Montenegro - The construction of principal spectra curves for Lane-Emden systems and applications, Ann. Scuola Norm. Sup. Pisa Cl. Sci. 29 (2000), 193-229.

[36] P. Pucci, J. Serrin - The maximum principle, Birkhäuser Verlag, Basel, 2007.

[37] J. Serrin, H. Zou - Existence of positive entire solutions of elliptic Hamiltonian systems, Comm. Partial Differential Equations 23 (1998), 577-599.

[38] G. Sweers - Strong positivity in $C(\bar{\Omega})$ for elliptic systems, Math. Z. 209 (1992), 251-271.

[39] P. Tolksdorf - On the Dirichlet problem for quasilinear equations in domains with conical boundary points, Comm. P.D.E., 8 (1983), 773-817.

[40] P. Tolksdorf - Regularity for a more general class of quasilinear elliptic equations, J. Differential Equations, 51 (1984), 126-150.

[41] J. L. Vázquez - A strong maximum principle for some quasilinear elliptic equations, Appl. Math. Optim., 12 (1984), 191-202. 